\title{
Long-term imaging of calcium dynamics using genetically encoded calcium indicators and automatic tracking of cultured cells
}

\author{
Igor Razlivanov¹, Teresa Liew ${ }^{1}$ Eira Watts Moore¹, Alaa Al-Kathiri¹, Tayma Bartram¹,2, Dmitriy \\ Kuvshinov ${ }^{3}$ \& Anton Nikolaev*,1 \\ ${ }^{1}$ Department of Biomedical Sciences, The University of Sheffield, Western Bank, Sheffield, S10 2TN, UK, \\ 2Department of Oncology and Metabolism, The University of Sheffield, Medical School, Beech Hill Road, \\ Sheffield, S10 2RX, UK, ${ }^{3}$ Department of Chemical Engineering, University of Hull, Cottingham Road, Hull, \\ HU6 7RX, UK
}

BioTechniques 65: 37-39 (July 2018) 10.2144/btn-2018-0024

Keywords: automatic cell tracking • calcium dynamics $\bullet$ cultured cells $\bullet$ GCaMP5

Calcium dynamics is crucial for many signaling pathways and cell functions. Understanding how calcium regulates cell function often requires long-term imaging of calcium dynamics. Here we report a methodological approach of long-term (5-10 h) imaging of calcium dynamics in cultured cells. The approach links calcium imaging using genetically encoded calcium indicators and semi-automatic tracking of individual cells. It can be used in a large variety of situations, ranging from the role of calcium in biological processes to cell heterogeneity and screening of drugs modifying signaling pathways.

Many signaling pathways use calcium as a second messenger to co-ordinate cellular physiology in a multitude of processes including differentiation, cellular stress or apoptosis [1,2]. It is therefore crucial to understand how calcium dynamics influence these processes. Cultured cells are an excellent system to approach this problem due to their ease of use and accessibility to drug treatment or genetic manipulation. Signaling pathways involving calcium ions have been studied in detail using many cell lines, including cancer cell lines [3,4] and stem cells [5,6]. However, biological processes often occur over a scale of many hours or even days. Accordingly, there is a need for robust approaches to facilitate long-term imaging and quantification of calcium dynamics. Here we present an efficient and robust approach for long-term calcium imaging in cultured cells. This approach employs genetically encoded calcium indicators instead of calcium dyes [7] and uses semi-automatic cell detection and tracking of cultured cells.

The genetically encoded calcium indicator (e.g., GCaMP5) is expressed in HeLa cells under the control of a CMV promoter using standard lipofectamine 3000 transfection (Figure 1A). Cells are subsequently imaged using a wide field microscope (e.g., Nikon [Tokyo, Japan] Dual Cam Eclipse Microscope with perfect focusing system, 20x/0.75 objective, CMOS Andor [Belfast, Northern Ireland] Zyla cameras and 525-50 Green and 605-50 Red filters) with environmental control (37 degrees, $\mathrm{CO}_{2} / \mathrm{O}_{2}$ and humidity control). Agonists are manually added to the imaging chamber or automatically applied via peristaltic pump (e.g., Nemesys pump, Cetoni [Korbußen, Germany]) at the bottom of the imaging chamber and the total volume is regulated by a separate pump placed above the imaging solution. This experimental approach allows imaging of cytosolic calcium dynamics in cultured cells for more than $8 \mathrm{~h}$ (Figure 1B and [7]) without any detectable photobleaching or ectopic localization of GCaMP reporter within subcellular compartments. Longer imaging time may also be possible but may require better $\mathrm{CO}_{2} /$ $\mathrm{O}_{2}$ and humidity control and periodic application of fresh media.

A problem with conventional imaging of cells over long time periods is that cells often move around each other. To overcome this a Java-based cell analyzer (jCA) has been developed; a semi-automatic image analysis software that facilitates simultaneous tracking and calculation of average GCaMP5 fluorescence within individual cells (available at [8]). The jCA software can be manually adjusted for parameters including filter, background removal, and definition of minimal and maximal particle size (Supplementary data). The jCA uses a watershed algorithm cell segmentation and then connects regions of interest that overlap in neighbouring frames. Background subtraction using a rolling-

\section{METHOD SUMMARY}

A long-term imaging of calcium dynamics in cultured cells includes: 1) fluorescent imaging of calcium concentration in cultured cells using genetically encoded calcium indicators, and 2) semi-automatic segmentation and tracking of individual cells. It can be used in a large variety of situations, ranging from the role of calcium in biological processes to cell heterogeneity and screening of drugs modifying signaling pathways. 


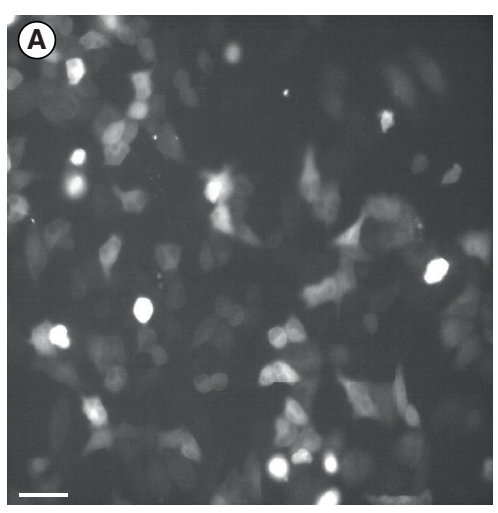

(B)
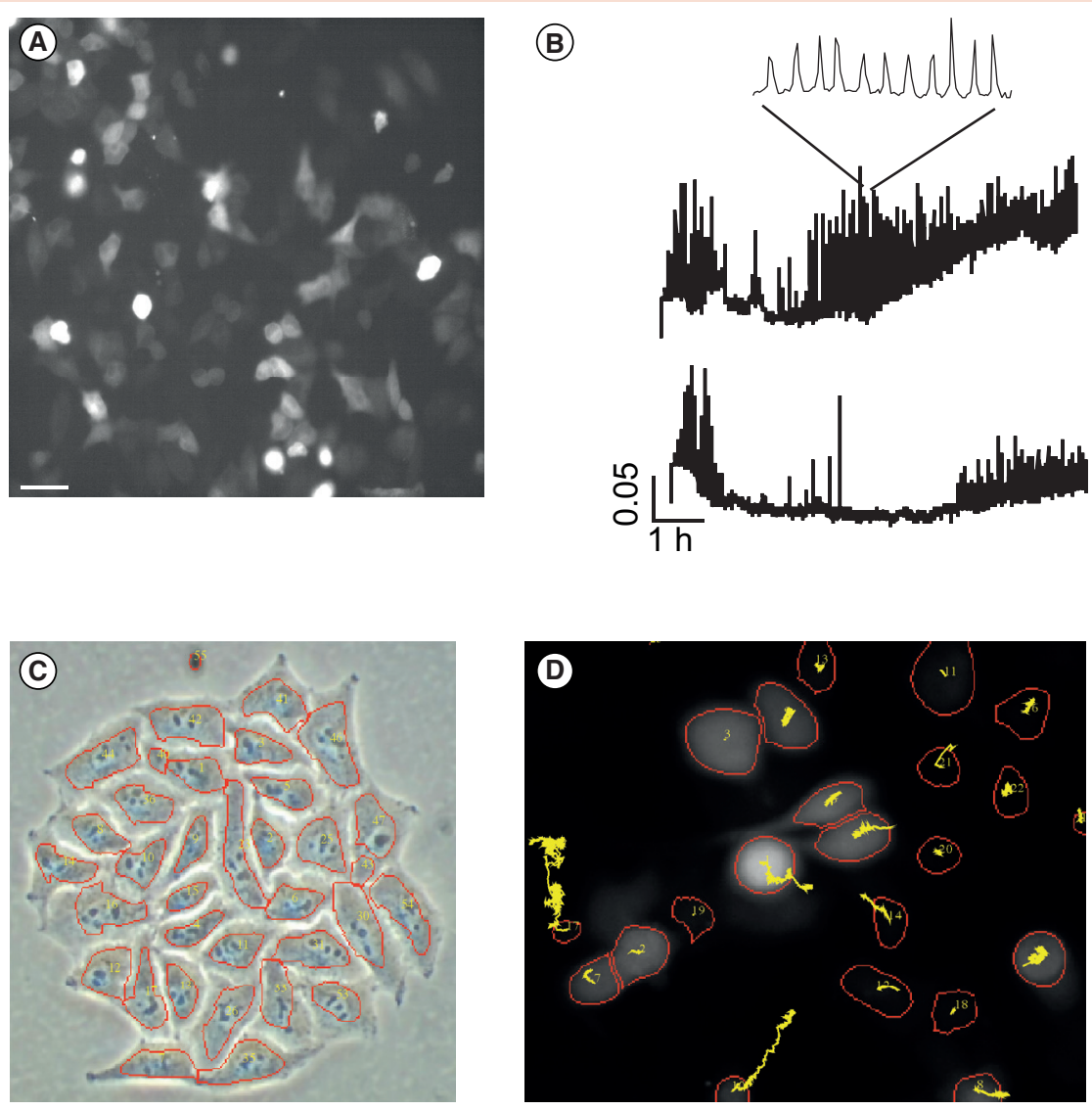

Figure 1. Long-term calcium imaging of HeLa cells using genetically encoded calcium indicators. (A) Example of HeLa cells transfected with CMV:GCaMP5 using lipofectamine 3000 transfection. Scale bar $=100 \mu \mathrm{m}$. (B) Examples of long-term calcium dynamics from cells shown in A. Inset on top shows expanded trace with calcium oscillations. Notice that there is no bleaching or decrease in the calcium reporter responsivity. Regions of interest were defined manually. (C, D) Cell segmentation using jCA software performed on phase-contrast (C) and red fluorescence (D). Cells were transfected with CMV:RFP construct).

ball algorithm enables optimal segmentation in a non-ideal background (e.g., dirt, glass scratches, etc.). Figure $1 \mathrm{C}$ shows an example of cell segmentation performed on phase-contrast images. On a standard dataset used in [9] and available at [10], the algorithm developed herein correctly identified $98.7 \%$ of cells $(n=1076$ cells from 11 images). For a movie of 2800 frames, the algorithm can extract full fluorescent dynamics of $20-50 \%$ of cells.

The basal cytosolic calcium concentration in many cell types is low, therefore, segmentation of cells based on calcium reporter fluorescence is not feasible. However, cells can be identified using phase-contrast images (Figure 1C) or alternatively, by co-expression of red fluorescent protein (Figure 1D). In this case, the jCA software defines the cell borders from the first reporter in the image set and reports the overall fluorescence from the second reporter (see Supplementary protocol).
Segmentation performed on phasecontrast and fluorescent images have their own advantages and disadvantages. Phase-contrast images are more uniform and therefore the parameters used for segmentation are similar for each individual cell. However, the segmentation on phase contrast images often results in segmentation of the nucleus area, rather than the entire cell, thus leaving some important cellular compartments not being analyzed.

Using this combined approach to chronic calcium imaging, we have recorded and analyzed the calcium dynamics of individual cells for many hours. Figure 2 shows an example and subsequent analysis of calcium oscillations reported in a large population of cells. Figure 2B demonstrates three examples of individual cells with distinct calcium dynamics: some of these cells exhibit calcium oscillations (Figure $2 \mathrm{~B}$, top), while others display no calcium oscillations (Figure 2B, bottom).
The resulting individual traces are then used to define the basic properties of calcium dynamics in individual cells and the entire population. One of the key properties of calcium oscillations that define their downstream output is their frequency [11]. Many cells do not exhibit continuous oscillations but rather oscillate in bursts that last for tens of minutes/hours (e.g., Figure 2B). It is therefore beneficial to calculate the power spectrum of individual traces and define the characteristic frequencies, rather than calculating average frequency or interspike interval over the entire experiment. Figure $2 \mathrm{C}$ shows three examples of power spectra of the traces, shown in Figure 2B. The top two traces have pronounced peaks at around 10 and $20 \mathrm{mHz}$, respectively. In addition to characterization of the dominating frequency using power spectrum, it may be useful to understand whether this dominating frequency changes with time. This can be explored using Wigner transform [12], shown in Figure 2D. Both cells, shown on the top of Figure $2 \mathrm{C}$, tended to decrease their characteristic frequency of calcium oscillation with time.

Using this approach it is now possible to address a variety of biological problems concerning the functional role of calcium in numerous cell types. One such question is the diversity of calcium signaling mechanisms in individual cells. Figure 2E demonstrates an example of a systematic analysis of diversity of oscillation frequencies in a population of 272 cells imaged in one experiment. The spiking rate of individual cells was automatically defined by low-pass filtering and subsequent thresholding of individual traces (see supplementary protocol and supplementary materials). The distribution is broad with some cells showing no calcium oscillations, while others demonstrate high-frequency oscillations.

Our approach also enables correlation of calcium dynamics with biological processes. Two classical examples [1,2,13] of processes with characteristic calcium dynamics are mitosis and apoptosis. During mitosis, calcium dynamics are downregulated and cells display no oscillations (Figure 2F, top) while apoptosis (detected by morphological changes in the cell) is characterized by a significant increase in cytosolic calcium concentration and no spiking (Figure 2F, bottom).

This approach also enables the application of multiple agonists and/or analysis of the long-term effect of chemical compounds 


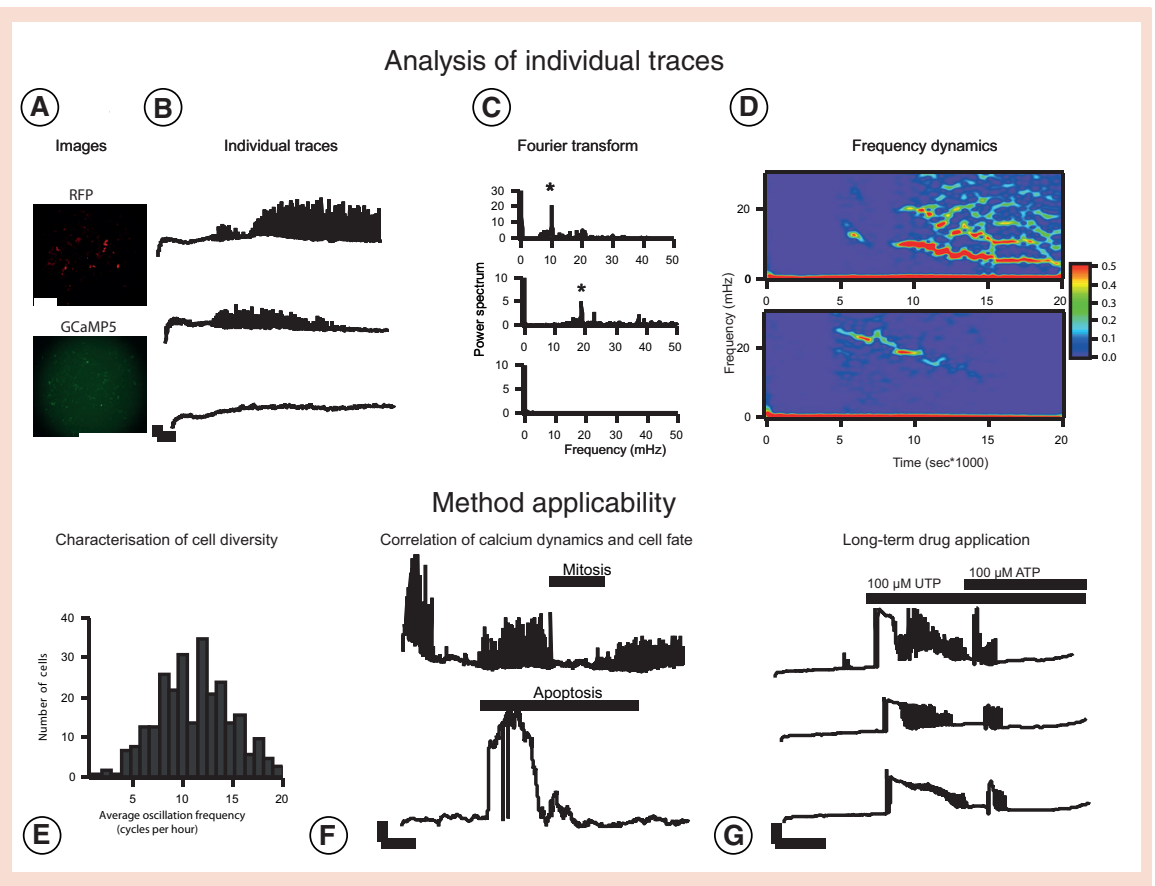

Figure 2. Analysis of calcium imaging data and examples of applications of the proposed approach. (A) Examples of cells transfected with RFP (top) and GCaMP5 (bottom) used for the analysis in Figure2. Scale bar $=200 \mu \mathrm{m}$. (B) Examples of calcium dynamics from three cells extracted using jCA software. Notice that the top two cells show calcium oscillations, while the bottom traces exhibit no calcium oscillations. Scale bars $=30 \mathrm{~min}$ and $0.05 \Delta \mathrm{F} / \mathrm{FO}$. (C) Power spectra of the three examples shown in $\mathrm{B}$. Notice that the top two traces exhibit characteristic oscillation frequency (10 and $20 \mathrm{mHz}$, respectively). (D) Wigner transforms performed on the top two traces shown in B and C. Notice that dominating frequency changes with time. (E) Distribution of average oscillation frequency in a population of 272 cells. (F) Example of calcium dynamics of cells undergoing mitosis (top) and apoptosis (bottom). Both mitosis and apoptosis, shown as black bars on top, were identified using changes in cell morphology. Scale bars $=1 \mathrm{~h}$ and $0.5 \Delta \mathrm{F} / \mathrm{FO}$. (G) Example of long-term application of calcium mobilizing agonists. Agonists were manually applied at $60 \mathrm{~min}$ and $120 \mathrm{~min}$ of the experiment. Scale bar $=30 \mathrm{~min}$ and $1 \Delta \mathrm{F} / \mathrm{FO}$.

on calcium dynamics. Figure 2G demonstrates examples in which the effect of UTP and ATP was assessed in the same cells for a prolonged period of time. Each compound was applied for $1 \mathrm{~h}$ and calcium dynamics of individual cells was defined.

Taken together, these results indicate that our approach enables better long-term imaging of calcium dynamics in individual cultured cells.

\section{Author contributions}

Anton Nikolaev, Igor Razlivanov and Dmitry Kuvshinov designed the study and wrote the paper. Anton Nikolaev, Teresa Liew, Eira Watts Moore, Alaa Al-Kathiri and Tayma Bartram performed the experiments. Igor Razlivanov and Anton Nikolaev performed the data analysis.

\section{Acknowledgements}

We thank Ryan MacDonald and Robert Wilkinson for the useful comments on the

\section{Supplementary data}

To view the supplementary data that accompany this paper please visit the website at: www.future-science.com/doi/ suppl/10.2144/btn-2018-0024

\section{References}

1. Ioshii SO, Yoshida T, Imanakayoshida K, Izutsu K. Distribution of a $\mathrm{Ca}^{2+}$ storing site in Ptk2 cells during interphase and mitosis - an immunocytochemical study using an antibody against calreticulin. Eur. J. Cell Biol. 66, 82-93 (1995).

2. Mattson MP, Chan SL. Calcium orchestrates apoptosis. Nat. Cell Biol. 5, 1041-1043 (2003).

3. Bootman M, Niggli E, Berridge M, Lipp P. Imaging the hierarchical $\mathrm{Ca}^{2+}$ signaling system in HeLa cells. J. Physiol. 499, 307-314 (1997).

4. Bootman MD, Berridge MJ. Subcellular $\mathrm{Ca}^{2+}$ signals underlying waves and graded responses in HeLa cells. Curr. Biol. 6, 855-865 (1996).

5. Kawano S, Otsu K, Kuruma A et al. ATP autocrine/ paracrine signaling induces calcium oscillations and NFAT activation in human mesenchymal stem cells. Cell Calcium 39, 313-324 (2006).

6. Kawano S, Shoji S, Ichinose S, Yamagata K, Tagami M, Hiraoka M. Characterization of $\mathrm{Ca}^{2+}$ signaling pathways in human mesenchymal stem cells. Cell Calcium 32, 165-174 (2002).

7. Garcia MI, Chen JJ, Boehning D. Genetically encoded calcium indicators for studying long-term calcium dynamics during apoptosis. Cell Calcium 61, 44-49 (2017).

8. Cell segmentation and tracking software for long-term calcium imaging. https://github.com/ tonza17/jCA

9. Arteta C, Lempitsky V, Noble JA, Zisserman A Learning to detect cells using non-overlapping extremal regions. Lect. Notes Comput. Sc. 7510, 348-356 (2012).

10. Cell systems dynamics group. www.bsse.ethz. ch/csd/software/faster.htm

11. Tompa P, Toth-Boconadi R, Friedrich P. Frequency decoding of fast calcium oscillations by calpain. Cell Calcium 29, 161-170 (2001).

12. Eggermont JJ, Smith GM. Characterizing auditory neurons using the Wigner and Rihacek distributions: a comparison. J. Acoust. Soc. Am. 87, 246-259 (1990).

13. Kapur N, Mignery GA, Banach K. Cell cycledependent calcium oscillations in mouse embryonic stem cells. Am. J. Physiol. Cell Physiol. 292, C1510-C1518 (2007).

zation or entity with a financial interest in or financial conflict with the subject matter or materials discussed in the manuscript apart from those disclosed.

No writing assistance was utilized in the production of this manuscript.

\section{Open access}

This work is licensed under the Creative Commons Attribution 4.0 License. To view a copy of this license, visit http://creativecommons.org/licenses/by/4.0/
First draft submitted: 28 March 2018; Accepted for publication: 28 May 2018

Address correspondence to: Anton Nikolaev; Department of Biomedical Sciences, The University of Sheffield, Western Bank, Sheffield, S10 2TN, UK E-mail: a.nikolaev@sheffield.ac.uk

To purchase reprints of this article contact: s.cavana@future-science.com 